\title{
Osteopenia of prematurity: Are we on the right track?
}

\author{
Sandya Bandara ${ }^{1}$, Amila Kariyawasam ${ }^{2}$ \\ Sri Lanka Journal of Child Health, 2012; 41(2): 56-59
}

(Key words: Osteopenia of prematurity)

\section{Background}

Modern advances in the care of preterm infants have significantly reduced the neonatal mortality rate universally. Among the Southeast Asian countries, Sri Lanka indicates the lowest neonatal mortality rate at $8.6 \%$ per 1000 live births. This achievement brings to attention the need to not just ensure the survival of the preterm infant but also the requirement to maintain better long term health outcomes and to manage associated co-morbidities relating to such newborn babies. Osteopenia of prematurity (OOP) in preterm infants is an important co-morbidity in its acute stage as well as long term as it is known to affect linear growth and lead to osteoporosis in patients in later life.

Premature infants are considered to be at high risk for developing osteopenia due to their reduced bone mineral content (BMC). Currently there are no standard practices relating to the screening, diagnosis or treatment of OOP around the world. At the neonatal intensive care unit (NICU) at Peradeniya Teaching Hospital in Sri Lanka we have observed clinical and radiological evidences of bony fractures due to reduced BMC which is very rare even in extremely premature babies. Most of the preterm infants in our unit were fed exclusively breast milk and parenteral nutrition was given only in selected cases.

Approximately $50 \%$ of very low birth weight (VLBW) neonates develop OOP with rickets which may eventually lead to spontaneous or traumatic fractures ${ }^{1}$. In the acute neonatal phase, reduced BMC can lead to fractures, which have been described in up to $10 \%$ of low birth weight (LBW) infants $^{2}$. The clinical onset of OOP is usually between 6 and 12 weeks postnatally. Neonates who have suffered intrauterine growth retardation (IUGR) are also prone to reduced stores of calcium secondary to decreased placental nutrient transfer ${ }^{3}$. Compared with mature newborns at birth, premature babies have larger marrow cavities and lower bone density when they have reached expected term ${ }^{4}$. Rickets in newborns was first described in a study conducted in 1919. All 668

${ }^{1}$ Consultant Paediatrician, ${ }^{2}$ Medical Officer, NICU, Teaching Hospital Peradeniya low-birth-weight infants studied at the time were found to have rickets 5 . There are several biochemical investigations, such as serum calcium, serum phosphate and alkaline phosphatase (ALP) that have been used to act as markers of bone mineralization and popular in screening for OOP.

\section{Risk factors}

The majority of bone mineralisation, along with calcium and phosphate accretion occurs during the third trimester of pregnancy. Infants born before the third trimester have decreased levels of these minerals. There are added factors that compound the risk of reduced bone mineralisation following premature delivery. There is evidence that the placenta has a role in BMC. Vitamin D is converted to 1, 25-dihydrocholecalciferol in the placenta which is important in the transfer of phosphate across the placenta to the fetus ${ }^{6}$.

There is an association between neonatal rickets and pre-eclampsia ${ }^{7}$. Severe demineralisation has also been described in infants born to mothers with chorioamnionitis and placental infection ${ }^{8}$. There is a higher incidence of postnatal rickets in babies with IUGR, suggesting that chronic damage to the placenta may alter phosphate transport ${ }^{9}$. VLBW, delayed establishment of full enteral feedings and long term use of parenteral nutrition are risk factors for OOP. In exclusively breast fed infants OOP does not depend on the severity of the prematurity ${ }^{10}$. Common neonatal conditions such as sepsis, cerebral pathology, muscular disorders and paralysis may result in prolonged periods of immobility, well recognized as a risk factor for poor bone mineralization. Conditions such as bronchopulmonary dysplasia, cholestasis, acidosis and necrotizing enterocolitis are other well known risk factors. Medications frequently used such as steroids, methylxanthines and diuretics, can increase the risk of inadequate bone mineralisation.

\section{Serum biochemical markers}

Alkaline phosphatase (ALP)

ALP is a glycoprotein enzyme produced by a variety of tissues including bone, liver, kidney and intestine. Tissue nonspecific ALP is generally measured; however, $90 \%$ of ALP in infants is 
known to be of bone origin and it is considered to be an indicator of bone turnover. During the first 23 weeks after the birth of all newborn babies ALP increases and further rises if there is a lack of mineral supply. If there is an abnormal increase in bone activity due to factors such as rapid growth or a deficiency in mineral content a biochemical indicator can be of assistance in the diagnosis of osteopenia in newborn babies. However, there seems to be insufficient evidence and conflicting theories as to whether ALP is such an indicator. An ALP level of greater than five times the upper adult limit of normal is an indicator of the risk of rickets ${ }^{11}$. By using dual energy $\mathrm{x}$-ray absorptiometry (DEXA) scan as a screening tool, Faerk et al concluded that bone mineralisation in premature infants cannot be predicted from serum ALP or serum phosphate ${ }^{12}$. However, ALP is still widely used because it is a readily available measurement and can be easily followed. Thus it is a frequently used screening tool for OOP which can generally be suspected when there is elevated ALP activity (>800 IU).

\section{Calcium}

Serum calcium cannot be regarded as a suitable method of screening as it can indicate normal values even though there may be a loss of bone calcium. The serum calcium level can also rise due to phosphorus depletion and hypophosphataemia.

\section{Phosphate}

Data has confirmed that although phosphate concentration is related to bone mineral density, it is not sensitive enough to identify infants with bone mineral deficits. It is however highly specific ${ }^{13}$. The use of serum phosphate levels in combination with ALP levels can significantly increase the sensitivity of the screening and identification of infants at risk of metabolic bone disease ${ }^{14}$.

\section{Radiological investigations}

$X$-ray

Osteopenia can be discovered as an incidental finding on a plain radiograph, showing 'thin bones' or healing fractures. Bone mineralization, however, needs to be decreased by at least $20-40 \%$ for these changes to be visible ${ }^{15,16}$

\section{Single photon absorptiometry (SPA)}

Data from SPA studies measuring BMC has been used to construct curves of intrauterine BMC. Such curves can subsequently be applied in longitudinal studies to compare the BMC in LBW infants with that of infants born closer to term ${ }^{17}$. Using this technology, many studies have documented delays in bone mineralisation in premature infants compared with the intrauterine curve of mineral accretion.
Dual photon absorptiometry (DPA)

DPA is a technique for quantifying both total skeletal and soft tissue masses. DPA is not used much in neonates due to time factors relating to the completion of a scan. DPA can take 20 to 50 minutes for a scan whereas SPA takes less than 5 minutes and therefore is considered a preferred method.

Dual-energy $x$-ray absorptiometry (DEXA)

It is becoming more widely used in infants but availability is limited. DEXA holds more promise for measuring $\mathrm{BMC}$ and assessing osteopenia in premature infants. DEXA uses two electronically generated x-ray beams of relatively high and low energy levels and can determine total body BMC as well as regional BMC with minimal tissue radiation. However, the scan time is still relatively lengthy (about 15 min for total body BMC), and the equipment is too cumbersome to be used at the bedside for studies of sick premature infants. Only a few studies using this technique in premature infants have been published and no standard reference values are available for preterm infants of different gestational ages ${ }^{18}$. DEXA scans are sensitive in detecting small changes in BMC and density, and can predict risks of fractures ${ }^{19}$.

\section{Quantitative ultrasound}

Ultrasound gives measurements that are related to bone mineral density and structure. It is simple, non-invasive, and a relatively cheap bedside test ${ }^{20}$. Reference values are available for both term and preterm infants ${ }^{21}$.

\section{Urine analysis}

Urinary excretion of calcium and phosphorus Fetal bone mineral accretion has been achieved in preterm infants by establishing and maintaining a simultaneous slight excretion of calcium and phosphorus (urine concentrations of $1-2 \mathrm{mmol} / \mathrm{l}$ ) by means of an individual supplementation with calcium and/or phosphorus, resulting in a slight surplus supply. However, to date it has not been proven that these variables are a reliable substitute for direct measurement of $\mathrm{BMC}^{22}$. Phosphate is not bound in the plasma like calcium and so the percent tubular reabsorption of phosphate (TRP) is the best guide to adequacy of phosphate supplementation. A percent tubular reabsorption of $>95 \%$ shows inadequate supplementation.

\section{Current situation in Sri Lanka}

We have collected the data using a standard questionnaire from 13 major neonatology units in Sri Lanka viz. Castle Street Hospital, De Soysa Maternity Hospital, Teaching Hospital Kalubowila, Sri Jayawardenepura General Hospital, Teaching 
Hospital Karapitiya, Teaching Hospital Kandy, Teaching Hospital Kurunegala, Teaching Hospital Kegalle, Teaching Hospital Jaffna, General Hospital Ratnapura, Teaching Hospital Anuradhapura, General Hospital Ampara and General Hospital Polonnaruwa. Out of these only four units screened for OOP and all four units used serum ALP as the screening method and one unit used x-rays of both wrist joints in extremely premature babies to confirm the diagnosis. Only one unit did monitoring of ALP every 2 weeks while the other 3 units did it monthly. The values of ALP taken as positive varied from 400IU/L$1000 \mathrm{IU} / \mathrm{L}$. Treatment was started guided by the maturity of the neonate where the three units had different borderlines like 30 weeks, 32 weeks and 36 weeks irrespective of their weight. Most of the units started treatment at 2 weeks in premature babies thought to be at high risk. For treatment all the units used calcium supplements and vitamin D. None of the units used phosphate supplements.

Our survey showed that in Sri Lanka we do not have proper diagnostic criteria to diagnose OOP. Further, there is no clear opinion about when and what to use for treatment. However, the survey showed that the most popular and freely available screening tool for OOP is ALP as all three units used it for screening.

Calcium (Ca) content of breast milk rises from about $4 \mathrm{mmol}(160 \mathrm{mg} / \mathrm{L})$ in initial colostrum to $6.4 \mathrm{mmol}(256 \mathrm{mg} / \mathrm{L})$ by the third day and plateaus there for the first three months of nursing ${ }^{23}$. Milk from mothers giving birth prematurely ("preterm milk") is not significantly different in Ca content from term milk during the first month of nursing ${ }^{24}$.

Recommended daily $\mathrm{Ca}$ intake is 140-160 mg $/ 100 \mathrm{kcal}$ and for phosphate $95-108 \mathrm{mg} / 100 \mathrm{kcal}$ (American Academy of Paediatrics) ${ }^{13}$. Recommended daily intake of vitamin $\mathrm{D}$ for premature infants is 400IU. When mothers and their infants are exposed to biologically normal amounts of sunlight, human milk must be assumed to contain biologically normal amounts of vitamin $\mathrm{D}^{25}$.

With the results of our survey we got to know that most of the units do not screen for OOP, and treatment is also not appropriate. After reviewing the current literature, in view of improving the screening and treatment methods of OOP we suggest the following guidelines:

Premature infants should be monitored for OOP if:

1. Period of gestation $<34$ weeks

2. Birth weight $<1800 \mathrm{~g}$
Monitoring:

This should include weekly serum ALP, calcium (Ca), and phosphorus (P) (Normal values of ALP $<800 \quad \mathrm{IU} / \mathrm{L}, \quad \mathrm{Ca}-\quad 2.26-2.69 \mathrm{mml} / \mathrm{L}, \quad \mathrm{P}-1.74-$ $2.66 \mathrm{mml} / \mathrm{L}$ )

Suggested treatment:

1. Calcium $140-160 \mathrm{mg} / \mathrm{Kg}$ daily

2. Phosphate $1-2 \mathrm{mmol} / \mathrm{Kg}$ daily

3. Vitamin D 400IU daily

\section{References}

1. Moyer-Mileur. Physical activity and diet: Key components for improved bone mass in premature, very low birth weight infants. Pediatric Research 1999; 45:287A.

2. Dabezies EJ, Warren PD. Fractures in very low birth weight infants with rickets. Clin Orthop Relat Res 1997; 335: 233-9.

3. Wahlig TM, Georgeiff MK. The effects of illness on neonatal metabolism and nutritional management. Clin Perinatol 1995; 22(11):7796.

4. Rauch F, Schoenau E. The developing bone: slave or master of its cell and molecules? Pediatric Research 1990; 144:36-40.

5. Backstrom M, Kuusela A, Maki R. Metabolic bone disease of prematurity. Annals of Medicine 1996; 28: 275-82.

6. Weisman Y, Harell A, Edelstein S, et al. 1 alpha, 25-Dihydroxyvitamin D3 and 24,25dihydroxyvitamin D3 in vitro synthesis by human decidua and placenta. Nature 1979; 281: 317-9.

7. Bosley AR, Verrier-Jones ER, Campbell MJ. Aetiological factors in rickets of prematurity. Arch Dis Child 1980; 55:683-6.

8. Lucas A, Brooke OG, Baker BA, et al. High alkaline phosphatase activity and growth in preterm neonates. Arch Dis Child 1989; 64: 902-9.

9. Holland PC, Wilkinson AR, Diez J, et al. Prenatal deficiency of phosphate, phosphate supplementation, and rickets in very-low-birth weight infants. Lancet 1990; 335: 697-701.

10. Sandya Bandara, Amila Kariyawasam, Incidence of osteopenia of prematurity in preterm infants who were exclusively fed 
breast milk. Early Human Development 2010; 86:S18

11. Kovar I, Mayne P, Barltrop D, et al. Plasma alkaline phosphatase activity: a screening test for rickets in pre term neonates. Lancet 1982; 1: $308-10$.

12. Faerk J, Peitersen B, Petersen S, et al. Bone mineralization in premature infants cannot be predicted from serum alkaline phosphatase or serum phosphate. Arch Dis Child 2002; 87: F133-6.

13. Harrison CM, Johnson $\mathrm{K}$, McKechnie E. Osteopenia of prematurity: a national survey and review of practice. Acta Paediatr 2008; 97(4):407-13.

14. Backstrom MC, Kouri T, Kuusela A-L, et al. Bone isoenzyme of serum alkaline phosphatase and serum inorganic phosphate in metabolic bone disease of prematurity. Acta Paediatr 2000; 89: 867-73.

15. Ardran GM. Bone destruction not demonstrable by radiography. $\mathrm{Br} J$ Radiol 1951; 24: 107-9.

16. Mazess RB, Peppler WW, Chesney RW, et al. Does bone measurement of the radius indicate skeletal status? J Nucl Med 1984; 25: 281-8.

17. Minton SD, Steichen JJ, Tsang RC. Intrauterine bone mineral content curves comparison with post natal bone mineral content curves. J Pediatr 1979; 95:1037-42.
18. Arora R, Arya S, Chellani H. Osteopenia of prematurity: a radiological perspective. Journal of Neonatology 2008; 22 (4): 249-53.

19. Syed Z, Khan A. Bone densitometry: applications and limitations. J Obstet Gynaecol Can 2002; 24: 476-84.

20. Rubinacci A, Moro GE, Boehm G, et al. Quantitative ultrasound for the assessment of osteopenia in preterm infants. Eur $J$ Endocrinol 2003; 149: 307-15.

21. Littner Y, Mandel D, Mimoumi FB, et al. Bone ultrasound velocity curves of newly born term and preterm infants. $J$ Pediatr Endocrinol Metab 2003; 16: 43-7.

22. Pohlandt F, Mihatsch WA. Reference values for urinary calcium and phosphorus to prevent osteopenia of prematurity. Pediatr Nephrol 2004; 19(11):1192-3.

23. Atkinson, S. A. (1995) Effects of gestational stage at delivery on human milk components. Jensen, R. G. eds. Handbook of Milk Composition 1995:222-237

24. Atkinson, S. A., Radde, I. C., Chance, G. W., Bryan, M. H. \& Anderson, G. H. (1980) Macro-mineral content of milk obtained during early lactation from mothers of premature infants. Early Hum. Dev. 4:5-14.

25. Mojab CG. Sunlight deficiency and breastfeeding. Breastfeeding Abstracts 2002; $22(1): 3-4$. 